COMMUNICATIONS IN

ANALYSIS AND GEOMETRY

Volume 9, Number 2, 351-367, 2001

\title{
Finiteness Results for Heegaard Surfaces in Surgered Manifolds
}

\author{
Yo'AV RIECK AND ERIC SEDGWICK ${ }^{1}$
}

\begin{abstract}
We demonstrate that for all but a finite number of Dehn fillings on a cusped manifold, the core of the attached solid torus is isotopic into every Heegaard surface for the filled manifold. Furthermore, if the cusped manifold does not contain a closed, non-peripheral, incompressible surface, then after excluding the aforementioned set and those filled manifolds containing incompressible surfaces (also a finite set) every other manifold obtained by Dehn filling contains at most a finite number of Heegaard surfaces that are not Heegaard surfaces for the cusped manifold. It follows that these manifolds contain a finite number of Heegaard surfaces of bounded genera. For each cusped manifold, the excluded manifolds are contained in a finite set that can be determined algorithmically.
\end{abstract}

\section{Introduction.}

This paper continues the authors' investigation into the Heegaard structure of manifolds obtained by Dehn filling [10]. Throughout, $X$ will denote a cusped manifold, i.e. an orientable 3-manifold with a single incompressible torus boundary component. Closed 3 -manifolds can be obtained by performing Dehn filling, attaching a solid torus to the boundary of $X$. It is straightforward to see that every Heegaard surface for $X$ will be a Heegaard surface for every manifold obtained by performing a Dehn filling on $X$. While it is possible that a filled manifold possesses a Heegaard surface that is not a Heegaard surface for $X$, here we are able to develop restrictions to this phenomenon.

Johannson (see [5]) has shown that a-toroidal Haken manifolds have

${ }^{1}$ The authors would like to thank the topology groups at Oklahoma State University, the University of California, Santa Barbara and the University of Melbourne for their hospitality during this research. Partial support was provided by the NSF through grant DMS95-10505/GIG. 
only finitely many Heegaard surfaces of bounded genera ${ }^{2}$ and Rubinstein [11] has shown that non-Haken manifolds contain at most a finite number of Heegaard surfaces of bounded genera. For non-Haken manifolds we achieve a weaker result, Corollary 5.2, with different methods. Our main objective is connecting all the Heegaard surfaces of a filled manifold to those of the manifold being filled as much as possible.

Pick a manifold $M$ obtained by filling $X$, and $\Sigma$ a Heegaard surface for it. If the core of the attached solid torus is not isotopic into $\Sigma$ the pair $(M, \Sigma)$ is called bad. The manifold $M$ is called bad if it contains some Heegaard surface $\Sigma$ so that $(M, \Sigma)$ is bad. A slope is called bad if the corresponding filled manifold is bad. It has been shown in [9] and [10] that good surfaces, i.e. Heegaard surfaces for $M$ into which the core is isotopic, are either Heegaard surfaces for $X$ or were obtained from such a surface by a single destabilization along the meridian. It is therefore desirable to rule out bad fillings as much as possible.

Indeed, the set of bad fillings was constrained in [7] and [9]. However, in both those works the authors had to assume the surfaces discussed had bounded genera. The main goal of this paper is to remove this assumption.

For convenience we will assume that $X$ is given via a one-vertex triangulation. (We can obtain a one-vertex triangulation by starting with a triangulation of $X$ for which all of the vertices are in the boundary [1], "closing the book" on any pair of adjacent triangles in the boundary which do not share all their vertices, and "layering on" additional tetrahedra when it is necessary to create additional book covers.)

Theorem 6.2. Let $\mathcal{T}$ be a one-vertex triangulation of the cusped manifold $X$. If $\Sigma \subset M$ is a bad Heegaard surface then the meridian of $M$ is either the slope of a boundary edge of the triangulation or the slope of a normal or almost normal surface in $(X, \mathcal{T})$.

In [4] it is shown that the set of slopes bounding normal or almost normal surfaces is finite, and of course there are only three boundary edges in a one vertex triangulation. Thus we obtain the following corollary:

Corollary 6.6. A cusped manifold $X$ has only finitely many bad slopes.

In other words, for all but a finite set of manifolds obtained by Dehn filling on a cusped manifold the core of the attached solid torus is isotopic

\footnotetext{
${ }^{2}$ Throughout this paper, "surfaces of bounded genera" means a collection of surfaces so that there exist an upper bound for their genera.
} 
into every Heegaard surface possessed by the filled manifold. Moreover, as a consequence of Corollary 6.6 and the work in [10], with the exception of the finite set of bad slopes and a (possibly infinite) set of slopes arranged along a finite number of lines in the Dehn surgery space, every Heegaard surface for a manifold in the Dehn surgery space is a Heegaard surface for $X$.

We then provide an application motivated by the examples of Casson and Gordon (see, e.g. [15]): pretzel knots that possess an infinite number of fillings producing manifolds each of which possesses an infinite number of strongly irreducible Heegaard surfaces (of arbitrarily high genera). Fundamental to this construction is the fact that $X$ contains an infinite number of essential spanning surfaces (of arbitrarily high genera). See the Examples in Section 4.

In fact, we demonstrate here that if a cusped manifold $X$ possesses an infinite number of fillings where the filled manifolds each have an infinite number of Heegaard surfaces which are not Heegaard surfaces for $X$, then $X$ possesses an infinite number of essential surfaces with boundary (each with at most 2 boundary components). In turn, this implies that $X$ also contains a closed non-peripheral essential surface.

A small manifold is one that does not possess a closed essential nonperipheral surface. For small manifolds we get:

Theorem 5.1. If $X$ is small then all but a finite number of manifolds in $D(X)$ contain at most a finite number of Heegaard surfaces that are not Heegaard surfaces for $X$.

The set of manifolds containing infinitely many Heegaard surfaces that are not Heegaard surfaces for $X$ is contained in a finite set that can be determined algorithmically.

Using Johannson's finiteness result (see above) we get:

Corollary 5.2. If $X$ is small then all but a finite number of manifolds in $D(X)$ have a finite number of Heegaard surfaces of bounded genera. The excluded manifolds are contained in a finite set that can be determined algorithmically.

This article is written in six sections: Sections 2 and 3 contain background material and definitions (some of which are new so the reader is encouraged to look over them). Sections 4 and 5 derive all the information necessary about "good" Heegaard surfaces, i.e. Heegaard surfaces for a filled manifold onto which the core of the attached solid torus can be isotoped. In Section 6 we treat bad Heegaard surfaces (i.e. Heegaard surfaces that are 
not good). This final section utilizes and assumes familiarity with Gabai's thin position argument and normal surface theory.

The authors would like to thank William Jaco, Hyam Rubinstein and Martin Scharlemann for inspiring conversations.

\section{Good, Bad and Other Basic Concepts.}

All surfaces and 3-manifolds considered are orientable and compact. We give the following definition of a Heegaard surface, and note that it is equivalent to the usual definition of a Heegaard surface as the common boundary of two compression bodies. For basic definitions see [12].

Definition 2.1. A closed, 2-sided surface $\Sigma$ embedded in a 3-manifold $M$ is called a Heegaard surface if $M$ can be constructed by attaching 2 and 3 handles to a product neighborhood, $\Sigma \times I$, of $\Sigma \subset M$.

A Heegaard surface is called stabilized if there exist two disks in its complement, meeting transversely in exactly one point. Otherwise the surface is said to be non-stabilized .

Throughout we will consider $X$ to be a cusped manifold, a 3-manifold with a single incompressible torus boundary component, denoted $T$. Our main tool for studying manifolds is via Dehn filling, i.e. attaching a solid torus to the cusped manifold $X$ along $\partial X$. The collection of all manifolds thus obtained is called the Dehn Filling Space, denoted $D(X)$. At times we will restrict our attention to small cusped manifolds, i.e. cusped manifolds that do not contain a closed non-peripheral incompressible surface. Dehn filling on a small cusped manifold will produce at most a finite number of manifolds that are Haken, i.e., contain a closed, two-sided, incompressible surface [3]. The results of this paper hold also when $X$ has more than one boundary component, and the Dehn fillings are performed along $T$, a distinguished incompressible boundary torus component. In that case, when referring to filled manifolds, Haken and non-Haken should be replaced by not small and small, respectively.

Since attaching a solid torus is equivalent to attaching one 2 -handle and one 3-handle, immediately from our definition of Heegaard surface we get that any Heegaard surface for $X$ is a Heegaard surface for any filled manifold. The converse is not true: for example the filled manifold may be $S^{3}$, and a Heegaard surface for it is $S^{2}$. This will never be a Heegaard surface for $X$. (Note that the core of the attached solid torus is never isotopic into this $S^{2}$.) 
From this point on, we will be looking for obstructions for such phenomena. The main concepts are defined below:

Definitions 2.2. $\quad$ 1. Let $M$ be a manifold obtained by filling $X$ and $\Sigma$ a Heegaard surface for it. The pair $(M, \Sigma)$ is called bad if the core of the attached solid torus is not isotopic into $\Sigma$.

2. A filling is called bad if the filled manifold contains a Heegaard surface $\Sigma$ so that $(M, \Sigma)$ is bad. Note that $\Sigma$ can be assumed to be nonstabilized.

3. The converse of bad is good.

4. A good Heegaard surface for $M \in D(X)$ is called non-trivial if it is non-stabilized and cannot be isotoped in $M$ to be a Heegaard surface for $X$.

\section{The Circular Logic of Destabilization Lines.}

The material in this section is not new and is presented here for completeness of presentation.

The good $\leftrightarrow$ bad dichotomy is essential to our study. In Section 6 we show that there are only finitely many bad fillings, and here we analyze good fillings. A secondary dichotomy appears when considering a good Heegaard surface $\Sigma \subset M \in D(X)$ : either $\Sigma$ is a non-trivial Heegaard surface or it is a Heegaard surface for $X$ (perhaps after isotopy in $M$ ). We are quite pleased when it is a Heegaard surface for $X$, and aim to place restrictions on the case when it is not.

There are two operations that we will need to perform with surfaces in a good manifold. First, if $\Sigma$ is a good Heegaard surface, we may isotope the core of the attached solid torus into $\Sigma$ and drill it out. The resulting surface is properly embedded in $X$ and has two boundary components. By choice of isotopy we may be able to obtain more than one surface in this fashion, any one of these will be denoted by $\Sigma^{*}$. Conversely, given a surface $\Sigma^{*}$ with two boundary components that is properly embedded in $X$, we get a closed surface $\widehat{\Sigma^{*}} \subset M$ by "capping it off", i.e. by attaching to $\partial \Sigma^{*}$ one of the two annulus components of $T-\partial \Sigma^{*}$.

Definitions 3.1. 1. A twice punctured surface $\left(\Sigma^{*}, \partial \Sigma^{*}\right) \subset(X, T)$ is called almost Heegaard if $X$ can be constructed by thickening $\Sigma^{*} \cup T$ and attaching 2 and 3 handles, where no attachments are performed along $T$ (cf. Definition 2.1). 
2. Let $\alpha$ be a slope on $T$. By the line defined by $\alpha$ we mean the set of all slopes intersecting $\alpha$ once exactly.

3. An almost Heegaard surface $\Sigma^{*}$ above is called genuine if for some manifold $M$ on the line determined by the slope of $\Sigma^{*}$ the closed surface $\widehat{\Sigma^{*}}$ obtained by capping it off is a non-trivial Heegaard surface for $M$. (Recall Definition 2.2 \#4).

Consider an almost Heegaard surface $\left(\Sigma^{*}, \partial \Sigma^{*}\right) \subset(X, T)$. Cap it off to obtain $\widehat{\Sigma^{*}}$. Then $\widehat{\Sigma^{*}}$ is a Heegaard surface for any manifold obtained by filling along a slope which meets a component of $\partial \Sigma^{*}$ once (see [6]). If $\Sigma^{*}$ is a genuine almost Heegaard surface, by assumption $\widehat{\Sigma^{*}}$ is not a Heegaard surface for $X$. However, as Figure 3.1 suggests, after stabilizing $\widehat{\Sigma^{*}}$ once a Heegaard surface for $X$ is obtained. In the compression body not containing $T$ there is a properly embedded disk $D$ which runs along the attached handle in a single arc.

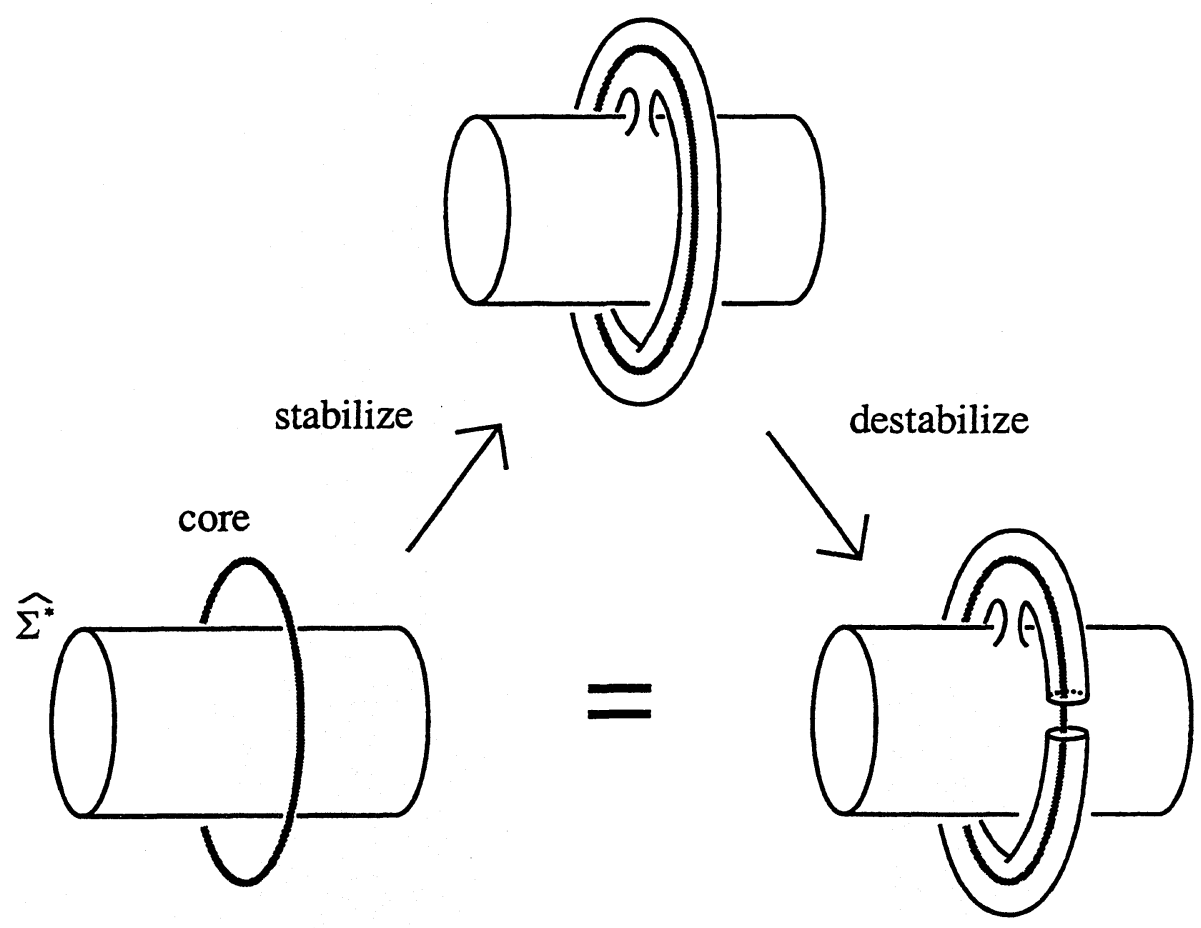

Figure 1: Stabilizing a good Heegaard surface for $M$ to get a Heegaard surface for $X$. 
Conversely, starting with a non-stabilized Heegaard surface $\Sigma$ for $X$ that possesses a disk $D$ as above ${ }^{3}$ there is a line of manifolds in $D(X)$ for which $\Sigma$ destabilizes, as the meridian intersects $D$ once. After such a filling, destabilize $\Sigma$. Clearly the core is isotopic into the destabilized surface. Isotope it into that surface and drill it out, obtaining $\Sigma^{*}$. Thus we obtain an almost Heegaard surface. Note that the slopes for which $\Sigma$ destabilized are precisely the slopes which meet $\Sigma^{*}$ once. We thus complete the circle, and motivate the following definition:

Definition 3.2. The line defined by the slope of a genuine almost Heegaard surface is called a destabilization line.

Remark. Let $M$ be a filled manifold that possesses a Heegaard surface $\Sigma$ that is not isotopic in $M$ to a Heegaard surface for $X$. According to our definitions at least one of the following holds:

1. $M$ is a bad manifold,

2. $M$ is a Haken manifold, or

3. $M$ is a good non-Haken manifold and therefore lies on a destabilization line defined by the slope of the genuine almost Heegaard surface $\Sigma^{*}$.

We show in Section 6 that there are only a finite number of manifolds of the first type. If $X$ is small then manifolds of the second type are finite in number. In the next section we show that although there may be an infinite number of manifolds of the third type, they are contained on a finite number of lines in the Dehn surgery space, $D(X)$.

\section{Incompressibility of Genuine Almost Heegaard Surfaces.}

Using Corollary 6.6, in studying Heegaard structure in the Dehn Surgery Space we do not study bad fillings (until Section 6), nor do we consider trivial good fillings.

As discussed above, a non-trivial good surface is a destabilization of a Heegaard surface for $X$, and the manifolds containing such surfaces are arranged along lines in $D(X)$. A priori, there can be infinitely many such

\footnotetext{
${ }^{3}$ We describe the important property of $D: D$ is an embedded disk in the compression body not containing $T$. There exists a second disk $D^{\prime}$ in the compression body $V$ containing $T$ so that $V$ cut open along $D^{\prime}$ contains a piece homeomorphic to $T^{2} \times I$ (where $T^{2}$ is the torus) and $D$ intersects that piece in a single arc.
} 
lines, and surfaces for $X$ may destabilize in many different ways (that is, one surface may destabilize in infinitely many ways yielding non-isotopic surfaces or infinitely many surfaces for $X$ may destabilize in one filling). In this section we develop a constraint to these phenomena.

\section{Examples.}

1. Morimoto and Sakuma have demonstrated that certain (toroidal) knot exteriors admit an infinite number of non-isotopic Heegaard surfaces of genus 2 [8]. In [13] Rubinstein and Scharlemann demonstrated that there is a bound on the genus of the common stabilization for every pair of these surfaces. It follows that there is a single surface that stabilizes this entire collection (recall that stabilization is unique). Therefore there must exist a surface that destabilizes in infinitely many ways.

2. The Casson-Gordon examples were constructed in manifolds arranged along a destabilization line in the Dehn Surgery Space of a certain pretzel knot. In each manifold on the line infinitely many Heegaard surfaces for the knot exterior destabilize, each yielding a non-stabilized Heegaard surface for the filled manifold. While it is possible that the destabilized Heegaard surfaces are isotopic in every one of the filled manifolds to a Heegaard surface for the knot exterior, this seems unlikely.

An essential ingredient in this construction is the fact that the pretzel knot exterior contains an infinite collection of free spanning surfaces of arbitrarily high genus. This implies the existence of a closed essential surface of genus at least 2 in the knot exterior (c.f. Theorem 5.1).

These examples provide evidence for the necessity of the assumption that $X$ is small in some of our statements.

We first place restrictions on destabilization lines. (This is a version of the work done in [10], simplified greatly by the assumption that the filled manifold is non-Haken, and with a stronger conclusion.)

Theorem 4.1. Let $X$ be a small cusped manifold, $M \in D(X)$ a non-Haken manifold, and $\Sigma \subset M$ a non-trivial good Heegaard surface.

Then $\Sigma^{*}$ is essential.

Remark. We may be able to isotope the core onto $\Sigma$ in more than one way, obtaining more than one surface that fits the description of $\Sigma^{*}$ above (see Section 3). Theorem 4.1 holds for any of them. 
Proof. Assume to the contrary that $\Sigma^{*}$ compresses. Denote by $F$ the surface obtain by compressing $\Sigma^{*}$ maximally. Since $M$ is an irreducible non-Haken manifold, the surface $\Sigma$ is strongly irreducible (it is not stabilized). Thus the compressing disks for $\Sigma^{*}$ form an embedded collection of disks $\Delta$ which lie only to one side of the surface $\Sigma^{*}$.

\section{Case 1: $F$ is essential.}

1. If $F$ is an almost Heegaard surface: cap $F$ off to obtain $\widehat{F}$. Then $\Sigma=\widehat{\Sigma^{*}}$ compresses down to $\widehat{F}$, a Heegaard surface for $M$. So the Heegaard surface $\widehat{F}$ was obtained by compressing the Heegaard surface $\Sigma$ along the disks $\Delta$ to only one side of $\Sigma$. But this implies that the Heegaard surface $\Sigma \subset M$ is a Heegaard surface for one of the compression bodies bounded by $\widehat{F}$, and is therefore, by Scharlemann and Thompson [14], a stabilization of $\widehat{F}$, contrary to our assumption.

2. Otherwise at least one of the components of $X$ cut open along $F$ is not a compression body, or it is but $\widehat{F}$ is not its attaching region. This component will contain a closed incompressible surface not parallel to $\partial X$, which remains essential in $X$ (since $F$ is essential), contradicting our assumption that $X$ is small.

Case 2: $F$ is inessential. Since $F$ is incompressible it must be a boundary compressible annulus. To complete the proof, we will argue that $\Sigma$ is trivial.

Let $D$ denote the boundary compressing disk for $F$. Since $\Sigma$ was a strongly irreducible Heegaard surface, the collection $\Delta$ was attached only to one side of $\Sigma^{*}$, and this must be the same side to which $D$ now lies. Tubing $F$ to retrieve $\Sigma^{*}$ we observe that $D$ is disjoint from the tubes. Hence $D$ is a boundary compressing disk for $\Sigma^{*}$, connecting distinct boundary components.

To show that $\Sigma$ is trivial, fill $X$ and push the core of the attached solid torus $\gamma$ into the side of $\Sigma$ that contains $D$. Cut $M$ open along $\Sigma$, denoting the side containing $D$ by $V$. Let $A$ be the annulus between $\gamma$ and $\Sigma$. Note that a neighborhood of $A$ union a neighborhood of $D$ form a solid torus of which $\gamma$ is a core, and this solid torus is attached to the rest of $V$ by a disk. Hence $\Sigma$ is a Heegaard surface for $X$, a contradiction.

From Hatcher ([3]) we get: 
Corollary 4.2. If $X$ is small then there are only finitely many destabilization lines in $D(X)$ that contain a non-Haken manifold with a non-trivial good Heegaard surface.

These destabilization lines are determined by slopes that are contained in a finite set that can be determined algorithmically.

Remark. We do not retrieve the stronger result of [10] which establishes finiteness of destabilization lines in general, as we do not consider filled manifolds which are Haken. It is possible that a destabilization line will be determined by a surface $\Sigma$ in a Haken manifold while $\Sigma$ is isotopic to a Heegaard surface for $X$ in the non-Haken manifolds on that line. No such example is known to us.

\section{Finiteness of Heegaard Surfaces for Most Non-Haken Manifolds.}

Using Corollary 6.6 we can now show:

Theorem 5.1. If $X$ is small then all but a finite number of manifolds in $D(X)$ contain at most a finite number of Heegaard surfaces that are not Heegaard surfaces for $X$.

The set of manifolds containing infinitely many Heegaard surfaces that are not Heegaard surfaces for $X$ is contained in a finite set that can be determined algorithmically.

Proof. We will exclude from consideration all manifolds in $D(X)$ that contain a bad Heegaard surface, an incompressible surface or are reducible. This is a finite set by Corollary 6.6 and [3].

It suffices to show that each of the remaining manifolds has but a finite number of non-stabilized Heegaard surfaces that are not Heegaard surfaces for $X$. This is because any stabilized Heegaard surface for a good filled manifold is also a Heegaard surface for $X$. To see this, destabilize the stabilized Heegaard surface, by assumption the core of the attached solid torus is isotopic onto the resulting Heegaard surface. Now stabilize this surface as shown in Figure 3.1, obtaining a Heegaard surface for $X$. This stabilization was unique (in $M$ ) and the resulting, i.e. the original, stabilized surface is also a Heegaard surface for $X$.

Now suppose that a filled manifold $M$ contains an infinite number of non-stabilized Heegaard surfaces that cannot be isotoped to be Heegaard 
surfaces for $X$. Isotope the core of the attached solid torus $\gamma$ into each of the surfaces. Drill $\gamma$ out to obtain $\left\{\Sigma_{i}^{*}\right\}_{i=1}^{\infty}$, an infinite collection of genuine almost Heegaard surfaces in $X$. Since $M$ is non-Haken, Theorem 4.1 implies that these are all essential.

As there are only finitely many slopes that bound essential surfaces, there exists a slope supporting infinitely many surfaces. This implies that the manifold $X$ contains a non-peripheral closed essential surface by using a standard normal surface argument. We give an outline of the argument here.

Choose least weight normal representatives of each of the essential surfaces. Since there are an infinite number of surfaces, one of these surfaces must be able to be expressed as the normal sum of another surface in the collection and a closed surface, $\Sigma_{i}^{*}=\Sigma_{j}^{*}+C$. Since the surfaces were taken to be least weight the closed surface $C$ is essential and non-peripheral. Moreover, if $\Sigma_{i}^{*}$ and $\Sigma_{j}^{*}$ are the same genus, then the closed surface is an essential torus, and if they have different genera, then it is an essential surface with genus at least 2 .

This implies finiteness of Heegaard structure of bounded genus for "almost all" manifolds, as Johannson proved this for Haken manifolds, which includes $X$. Since the cusped manifold $X$ is a-toroidal and Haken, it contains only a finite number of Heegaard surfaces of bounded genera [5]. Combining this with the previous theorem we have:

Corollary 5.2. If $X$ is small then all but a finite number of manifolds in $D(X)$ have a finite number of Heegaard surfaces of bounded genera. The excluded manifolds are contained in a finite set that can be determined algorithmically.

\section{Normal and Almost Normal Heegaard Surfaces.}

In this section we make extensive use of normal/almost normal surface theory and of thin position (see [2] for the basic concepts).

Let $\gamma$ be a knot in a manifold $M$. Let $\gamma_{p}$ be a knot embedded in the torus $\partial N(\gamma)$ so that the algebraic intersection of $\gamma_{p}$ with a meridian of $N(\gamma)$ is $\pm p$. Then we say that $\gamma_{p}$ is a $p$-cable of $\gamma$. So a 0 -cable of a knot is the meridian of $N(\gamma)$ and any 1-cable is ambient isotopic to $\gamma$ within $N(\gamma)$ (or a longitude). (A p-cable need not be unique, for example the $(5,1)$ and $(5,2)$ torus knots are distinct, but both are 5-cables of the unknot.)

Given a height function on a manifold and a knot embedded in it, the 
bridge number of the knot is the minimal number of minima (which equals minimal number of maxima) of the knot, taken over all Morse presentations. Bridge zero means the knot is isotopic into a level surface. Consistent with our definition from the previous sections, we call such a knot good, and a knot not isotopic into a level surface-bad. It is well known a Heegaard surface induces a height function (see [12]) on a manifold, and a knot is bad w.r.t. the Heegaard surface if and only if it is bad w.r.t. the induced height function.

Before going to the main theorem, we provide the following lemma:

Lemma 6.1. Let $\Sigma \subset M$ be a Heegaard surface which is bad with respect to a knot $\gamma \subset M$. Let $\gamma_{p}$ be a p-cable of $\gamma$. Then $\gamma_{p}$ has bridge number at least $p$ (with respect to the height function induced by $\Sigma$ ).

Proof. Let $A$ be the annulus defining the cable structure, i.e. an annulus with one boundary component running once along $\gamma_{p}$ and the other running $p$ times along $\gamma$.

Isotope $\gamma_{p}$ to be in minimal bridge position, and (subject to that constraint) isotope $\gamma$ to be in thin position. Since $\gamma$ is bad it cannot be isotoped into a level surface. A standard application of Gabai's thin position argument gives us a level surface, meeting $\gamma$, so that the intersection of the level surface with the annulus $A$ does not contain an arc that is boundary parallel in $A$ into $\gamma$ ( $c f$. [9], another application of thin position to the study of surfaces with boundary).

Thus every arc with an endpoint on $\gamma$ has the other endpoint on $\gamma_{p}$. As there are at least two intersections for $\gamma$ with the level surface, there will be at least $2 p$ arcs of intersection (one for each sheet of $A$ near $\gamma$ ) which implies that the bridge number of $\gamma_{p}$ is at least $p .^{4}$

This lemma gives us a criterion to detect when the core of the attached solid torus $\gamma$ is isotopic into a Heegaard surface (is good): if $\gamma_{p}, p \geq 1$, is isotopic into the Heegaard surface then so must be $\gamma$.

Remark (The Daisy Lemma). If $\Sigma$ is strongly irreducible, more is true: if the transverse intersection $\partial N(\gamma)$ and $\Sigma$ contains the curve $\gamma_{p}(p \geq 2)$,

\footnotetext{
${ }^{4}$ In fact, this argument gives us a little more: the bridge number of $\gamma_{p}$ is at least $p$ times the width of the thickest level of $\gamma$ in its thin position, but as we have no control over that number we phrased Lemma 6.1 as we did.
} 
then $\Sigma$ is a Heegaard surface for the knot exterior $X=M-N(\gamma)$. We shall not prove this surprising fact here.

In [16] Thompson gave a description of Rubinstein's recognition algorithm for $S^{3}$ (see [11]). Her argument was modified in [4] to provide a recognition algorithm for a knot exterior in $S^{3}$. Both of these arguments apply thin position to the genus 0 height function on $S^{3}$. Here we adapt these arguments to manifolds of higher genus. The essential ingredient added here is Lemma 6.1, which allows us to conclude that the intersection between the level surface and boundary torus is in meridional curves $(p=1)$, or the knot is good with respect to the Heegaard surface.

Endow $X$ with a triangulation $\mathcal{T}$ that has one vertex per boundary component. Then one vertex and three edges of the triangulation lie in $T$. We refer to the three edges in the boundary component $T$ as boundary edges, and note that each edge represents a slope in $T$. The vertices in $\partial X$ will be called boundary vertices. (Vertices and edges on $\partial M=\partial X-T$ play no special role.)

Theorem 6.2. Let $\mathcal{T}$ be a triangulation of the cusped manifold $X$ with one vertex per boundary component. If $\Sigma \subset M$ is a bad Heegaard surface then either

1. the meridian of $M$ is the slope of a boundary edge of the triangulation, -or-

2. the meridian of $M$ is the slope of a normal or almost normal surface in $(X, \mathcal{T}) .^{5}$

Proof.

Finding $e . \mathcal{T}$ restricted to $T$ consists of two triangles. After filling along $T$, $\mathcal{T}$ embeds in $M$. (Of course, $\mathcal{T}$ is not a triangulation of $M$, one component of the complement of $\mathcal{T}^{(2)}$ is a solid torus.) The Heegaard surface $\Sigma$ induces a height function $h: M \rightarrow[0,1]$, where $h^{-1}(0)$ and $h^{-1}(1)$ are embedded graphs union $\partial M$, and $h^{-1}(t)$ for $t \in(0,1)$ is a surface isotopic to $\Sigma$. The boundary vertex is now the unique vertex of $\mathcal{T}^{(0)}$ in the interior of $M$.

Put $\mathcal{T}^{(1)}$ (the one-skeleton of $\mathcal{T}$ ) in thin position w.r.t. $h$ subject to the condition that the boundary vertex is held at height 1 . To make use of thin position we need a boundary edge to have bridge number at least 2 .

\footnotetext{
${ }^{5}$ The boundary of this surface may also contain trivial curves.
} 
The boundary edges are cables of the core of the attached solid torus. By Lemma 6.1 , if one is a $p$-cable for $p \geq 2$ it must have bridge number 2 or more (by assumption the core is bad); assume all are $p$-cables for $p=0$ or $p=1$, i.e. each is a meridian or a longitude. It is not possible for all three to be longitudinal, so one must be meridional, this is the second conclusion of the theorem.

From this point on we shall assume that the meridian is not a boundary slope, and hence there exists a boundary edge with bridge number at least 2. Call it $e$.

Finding normal/almost normal surfaces. Near the minima (resp. maxima) of $\gamma$ bend the surfaces in $\mathcal{T}^{(2)}$ upwards (resp. downwards). Perturb $\mathcal{T}^{(2)}$ slightly so that $h \mid \mathcal{T}^{(2)}$ is Morse. A layer in $M$ is a subset of the form $h^{-1}([a, b])$, where $a$ and $b$ are consecutive critical points of $h \mid \mathcal{T}^{(1)}$. A layer is called thick if $b$ is a maximum of $h \mid \mathcal{T}^{(1)}$ and $a$ a minimum.

We show that every thick layer contains a normal/almost normal surface:

Since we bent $\mathcal{T}^{(2)}$ near the minima and maxima, applying the standard thin position argument yields a level surface between $a$ and $b$ that does not have a high disk nor a low disk. Hence all curves of intersection of that level with $\mathcal{T}^{(2)}$ are normal curves or are disjoint from the 1-skeleton.

Although Thompson proved the following lemmata for planar surfaces, the original proofs go through for a height function induced by a Heegaard surface of any genus. We omit them here.

In what follows we will pick a surface $\Sigma^{*}=\Sigma \cap X$, where $\Sigma=h^{-1}(t)$ for some $t$. (Our goal is to find the right value of $t$.) Let $\left(\Sigma^{*}, \partial \Sigma^{*}\right) \subset(X, T)$ be a level surface. The lemmata concern the intersection of $\Sigma^{*}$ with the tetrahedra of $\mathcal{T}$ :

Lemma 6.3 (Thompson). There does not exist a tetrahedron in $\mathcal{T}$ so that the intersection of $\Sigma^{*}$ with this tetrahedron contains a curve of length greater than 8.

Lemma 6.4 (Thompson). There does not exist a tetrahedron in $\mathcal{T}$ so that the intersection of $\Sigma^{*}$ with this tetrahedron contains two normal curves of length 8.

Lemma 6.5 (Thompson). There do not exist two distinct tetrahedra so that the intersection of $\Sigma^{*}$ with each of these tetrahedra contains a normal curve of length 8. 
If $\Sigma^{*}$ intersects a face of the triangulation in a simple closed curve compress it; within each tetrahedron compress it until it is a collection of disks. We may throw away any components that do not meet a pre-chosen edge.

The resulting surface is a normal or almost normal surface (using only an octagon, no tubes). We may require that it meets the edge $e$ if the original surface did.

Finding a normal/almost normal surface with an essential boundary component. We use the boundary edge that has bridge number greater than one, say $e$, to show that the intersection of $T$ with one of the normal/almost normal surfaces described above must contain an essential curve.

Assume the contrary: all boundary components of all normal/almost normal surfaces we found are inessential. Since $\mathcal{T}$ restricted to $T$ consists of two triangles, all normal curves of intersection that are inessential are vertex linking. The outermost of those bound a disk, name it $D$.

The edge $e$ consists of two arcs, $e_{1}=e \cap D$ and $e_{2}=c l\left(e-e_{1}\right)$. (That $e_{1}$ is a single arc follows from that fact that $\partial D$ is a normal curve.) If $e_{2}$ had both a minimum and a maximum, the procedure above would have yielded a normal/almost normal surface, with its boundary meeting int $\left(e_{2}\right)$. But by construction only points from $e_{1}$ can intersect these surfaces. So $e_{2}$ has only a single minimum (or maximum). Isotope $e_{1}$ into $\partial D$ (which is in a level surface) and perturb it to have a maximum (resp. minimum). Clearly $e_{1}$ has bridge number one in this presentation, a contradiction.

Using the surface. Let $\Sigma^{*}$ denote the level surface that intersects $\partial X$ in an essential curve. If the essential component of the boundary of $\Sigma^{*}$ is longitudinal $\gamma$ is good, contrary to our supposition. If it is not meridional or longitudinal it is some cable of $\gamma$. Since this cable lies on the Heegaard surface it has bridge number zero and by Lemma 6.1 we again get that $\gamma$ is good. Hence we conclude that it is a 0 -cable, i.e. it is meridional.

In [4], Jaco and Sedgwick proved that on $\partial X$ there are only finitely many slopes bounding almost normal surfaces. Add to this the three slopes of boundary edges. Together with this, Theorem 6.2 yields an analog to Hatcher's theorem [3] for Heegaard surfaces. These slopes are a subset of finite set that can be detected algorithmically.

Corollary 6.6. A cusped manifold $X$ has only finitely many bad slopes. 
Proof. Immediate.

\section{References.}

[1] R. H. Bing, Some aspects of the topology of 3-manifolds related to the Poincaré conjecture, Lectures on modern mathematics, Vol. II, 93-128, Wiley, 1964.

[2] D Gabai, Foliations and the Topology of Three-Manifolds III, J. Diff. Geom. 26 (1987), 479-536.

[3] A. E. Hatcher, On the Boundary Curves of Incompressible Surfaces, Pacific J. Math. 99 (1982), 373-377.

[4] W. Jaco and E. Sedgwick, Decision problems in the space of Dehn fillings, Submitted to Topology, Available at xxx.lanl.gov - math.GT/9811031, 1998.

[5] Klaus Johannson, Topology and combinatorics of 3-manifolds, SpringerVerlag, Berlin, 1995.

[6] W.B.R. Lickorish, A Representation of Orientable Combinatorial 3-Manifolds, Ann. of Math. 76 (1962), 531-540.

[7] Y. Moriah and J.H. Rubinstein, Heegaard Structures of Negatively Curved Manifolds, Comm. in Geom. and Ann. 5 (1997), 375-412.

[8] Kanji Morimoto and Makoto Sakuma, On unknotting tunnels for knots, Math. Ann. 289 (1991), 143-167.

[9] Yo'av Rieck, Heegaard structure of manifolds in the dehn filling space, to appear in Topology, 1997.

[10] Yo'av Rieck and Eric Sedgwick, Persistence of heegaard structure under dehn filling, preprint, 1997.

[11] J. H. Rubinstein. Polyhedral minimal surfaces, heegaard splittings and decision probiem for 3-dimensional manifolds, in Proceedings of the Georgia Topolgy Conference, 1997.

[12] M. Scharlemann, Hegaard splittings of compact 3-manifolds, to appear in Handbook of Geometric Topology.

[13] Hyam Rubinstein and Martin Scharlemann, Comparing Heegaard splittingsthe bounded case, Trans. Amer. Math. Soc. 350 (1998), 689-715.

[14] M. Scharlemann and A. Thompson, Thin position and Heegaard splittings of the 3-sphere, J. Diff. Geom. 39 (1994), 343-357. 
[15] E. Sedgwick, An infinite collection of Heegaard splittings that are equivalent after one stabilization, Math. Ann. 308 (1997), 65-72.

[16] A. Thompson, Thin position and the recognition problem for $S^{3}$, Math. Res. Lett. 1 (1994), 613-630.

RECEIVED MARCH 4, 1999.

UNIVERSITY OF MELBOURNE

PARKVILLE, ViCTORIA 3052

Australia

AND

OKLAHOMA STATE UNIVERSITY

STILLWATER, OK 74078

E-mail addresses: yoav@math.ucsb.edu

sedgwic@math.okstate.edu 